\title{
Über den Abbau von Aminosäuren im Organismus.
}

\author{
Von
}

Dr. L. Flatow.

(Aus der II. medizinischen Klinik in München.)

(Der Redaktion zugegangen am 21. Januar 1910.)

Das Schicksal der Eiweißsubstanzen im intermediären Stoffwechsel war bis vor kurzem in tiefes Dunkel gehüllt.

Zwar war bekannt, daß im Verdauungstractus ein weitgehender hydrolytischer Abbau der Proteine stattfindet - großenteils bis zu den Aminosäuren -; aber ob ein Wiederaufbau arteigenen Eiweißes in der Darmwand, in der Leber oder in den regenerationsbedürftigen Organen selbst vor sich geht, oder ob gar ein Teil der Proteintrümmer ohne vorherige Synthese zur Energieerzeugung verbrannt wird - etwa bei Eiweißüberernährung -, darüber fehlten bereits sichere Angaben.

Während so über die Art des Eiweißersatzes im Organismus auch jetzt noch kaum eine sicher gestellte 'Tatsache zu registrieren ist, hat die Erforschung der Vorgänge bei der Oxydation der Eiweißkörper in letzter Zeit einen wesentlichen Fortschritt erfahren.

Dieser Fortschritt ist einer Vereinfachung der Fragestellung zu danken.

Von der Vorstellung ausgehend, daß auch unter physiologischen Bedingungen im lebenden Organe zunächst hydrolytische Prozesse ablaufen, welche die vorhandenen Eiweißstoffe schrittweise in Aminosäuren überführen, und daß diese dann der Verbrennung unterliegen, ist nämlich von einigen Autoren (Neuberg u. Langstein, Blendermann, Blum, Neubauer) die komplizierte Frage der Eiweißoxydation auf die einfachere nach der Verbrennung der Aminosäuren reduziert worden.

Die so geschaffene Vereinfachung läuft allerdings einer noch nicht sicher widerlegten Hypothese zuwider, die Hofmeister sich über den Eiweißabbau gebildet hat.

Hof meister nimmt eine direkte Oxydation ganzer Peptidketten an; etwa, um sein Schema zu zitieren, in der Art, daß in diesem 


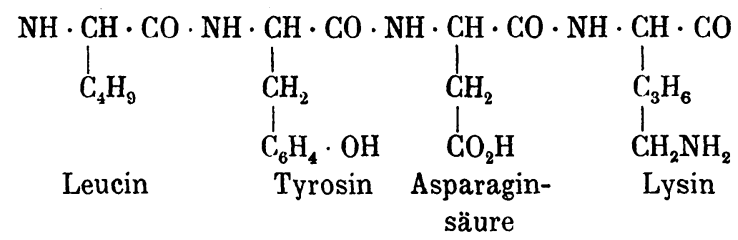

durch Verbrennung der Seitenketten eine aus Glycylresten bestehende Hauptkette gebildet wird, die ihrerseits oxydativ $\mathrm{CO}_{2}$ und Carbaminsäure liefert, mithin direkt einen Komplex des als Endprodukt auftretenden Harnstoffes.

Es lassen sich gegen die Hofmeister sche Theorie einige Bedenken nicht unterdrücken.

Bei der Cystinurie sind in manchen Fällen Aminosäuren im Harne gefunden worden, die also wohl auch im Blute vorhanden gewesen sein mußten. - Die Entstehung der Homogentisinsäure, die beim Alkaptonuriker als unvollkommenes Oxydationsprodukt des Tyrosins und Phenylalanins im Harn auftritt, ist gleichfalls unter Zugrundelegung der Hofmeisterschen Hypothese nicht verständlich.

Beweisend sind freilich diese Einwände nicht, da ihnen das Verhalten physiologisch abnormer Individuen zugrunde liegt.

Als Arbeitshypothese zum mindesten ist indessen die Vorstellung einer primären Hydrolyse der Proteine im Organismus uud die damit gegebene Vereinfachung der Fragestellung nach der Oxydation der Aminosäuren schon deswegen hinreichend begründet, weil das Endresultat der Verbrennung von Eiweiß und von Aminosäuren das gleiche ist. Beide gehen im wesentlichen in Harnstoff, $\mathrm{H}_{2} \mathrm{O}$ und Kohlensäure über.

Es haben sich verschiedene Autoren der Aufgabe unterzogen, nach Verfütterung von Aminosäuren im Harne nach stickstofffreien Substanzen $\mathrm{zu}$ suchen, die als intermediäre Abbauprodukte dieser zu deuten wären.

Die Verabreichung von physiologisch vorkommenden Aminosäuren hat indessen bisher ein klares Resultat nicht zu liefern vermocht.

Neuberg und Langstein ${ }^{1}$ ) verfütterten an hungernde

1) Neuberg u. Langstein, Verh. d. Berl. physiol. Ges., 1903. 
Kaninchen große Mengen Alanin und konnten darauf im Harne Milchsäure nachweisen.

Neubauer ${ }^{1}$ ) kritisiert diesen Befund; es könnten als Milchsäurebildner auch Kohlehydrate und Fette in Betracht kommen; auch habe Irisaw $a^{2}$ ) im Harne agonaler Kaninchen an sich Milchsäure nachweisen können. Die 10 Tage hungernden Kaninchen seien vielleicht agonal gewesen.

Als klar wird daher Neuberg und Langsteins Befund nicht zu betrachten sein.

Überhaupt stellen aliphatische Aminosäuren wegen der Vieldeutigkeit ihrer Abbauprodukte kein besonders gutes Versuchsmaterial dar.

Von aromatischen Aminosäuren hatte Blendermann ${ }^{3}$ ) schon früher an Kaninchen größere Quantitäten Tyrosin verfüttert.

Er konnte danach im Harne neben Tyrosinhydantoin<smiles>O=C1NC(=O)C(Cc2ccc(O)cc2)N1</smiles>

(also einem Paarungsprodukte des Tyrosins mit Carbaminsäure) noch eine zweite stickstofffreie Substanz isolieren, die er für p-Oxyphenylmilchsäure hielt.

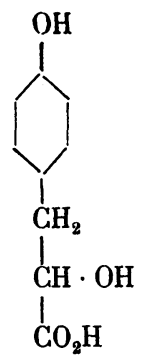

1) Neubauer, Habilitationsschrift 1908, München.

2) Iris awa, Diese Zeitschrift, Bd. XVII, S. 340.

3) Diese Zeitschrift, Bd. VI, S. 257.

Hoppe-Seyler's Zeitschrift f. physiol. Chemie. LXIV. 
Neubauer hat (l. c.) zur Kritik dieses Befundes die pOxyphenylmilchsäure dargestellt und fand die von ihm synthetisch erhaltene Substanz in Eigenschaften und Schmelzpunkt verschieden von der Blendermann schen Substanz; sie schmolz ca. $50^{\circ}$ niedriger wie diese, ein Verhalten, das selbst dann unwahrscheinlich wäre, wenn Blendermann optisch aktive Substanz in Händen gehabt hätte. Eine Angabe dārüber fehlt.

Es ist natürlich, daß bei ihrer Leichtverbrennlichkeit im Organismus physiologische Aminosäuren nur in Ausnahmefällen intermediäre Abbauprodukte im Harne erscheinen lassen werden.

Man hat weiterhin versucht, aus gelegentlich im Harne auftretenden Substanzen, deren Zusammenhang mit der Eiweißoxydation unzweifelhaft ist, Einblick in den Mechanismus des Aminosäureabbaus zu gewinnen.

Aus den oben angeführten Gründen haben speziell die aromatischen Säuren im Harn in dieser Hinsicht Deutungen erfahren.

Der Befund von p-Oxyhydrocumarsäure

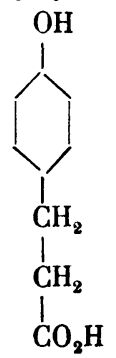

steht sicher in Beziehung zum Tyrosin.

Aber da sie aus diesem durch reduktive Ammoniakabspaltung entstanden sein müßte, und da sie ferner im Organismus unverbrennlich ist, stellt sie sicher kein intermediäres Abbauprodukt des Tyrosins dar. Vielmehr dankt sie Fäulnisvorgängen ihre Entstehung. Gleichfalls als Fäulnisprodukte erkannt, scheiden für die hier in Betracht kommenden Überlegungen p-Kresol, Indoxyl und ähnliche Phenole aus, die an Schwefelsäure gebunden im Harne auftreten. Die Abspaltung von Carboxylgruppen ist eine Fäulnisreaktion.

Von gelegentlich im Harne vorkommenden stickstofffreien 
aromatischen Substanzen ist schließlich noch das "Alkapton* zu erwähnen.

Es ist sichergestellt, daß bei der "Alkaptonurie» einer sehr seltenen familiären Stoffwechselabnormität im Harne eine Verbindung auftritt, deren Konstitution unzweifelhaft einer Hydrochinonessigsäure<smiles>O=C(O)CC1CC(O)CCC1O</smiles>

entspricht und die ebenso unzweifelhaft ihre Entstehung dem oxydativen Abbau des Tyrosins und Phenylalanins dankt.

Im Alkaptonurikerharn sollte sich ferner nach Kirk bisweilen eine zweite ähnliche Substanz vorfinden, die Uroleucinsäure genannt und von Baumann eingehend untersucht worden ist. Baumann sprach ihr mit einiger Wahrscheinlichkeit die Konstitution einer Hydrochinonmilchsäure zu.<smiles>O=C(O)C(O)CC1CC(O)CCC1O</smiles>

Aber wohl mit Unrecht, denn nach Untersuchungen von Ne ubauer und mir $^{1}$ ) ist die synthetische Hydrochinonmilchsäure in ihren Eigenschaften völlig different von der Uroleucinsäure.

Außerden hat Garrod und Hurtley ${ }^{2}$ ) bei einem von Kirk aufbewahrten und mit Uroleucinsäure bezeichneten Präparate feststellen können, daß es zum größten Teile aus Homogentisinsäure bestand.

Die Revue über alle jene im Harne auftretenden aromatischen Substanzen, die in Beziehung zum oxydativen Abbau des Eiweißes stehen, läßt so als einzigen Repräsentanten dieser Art die Homogentisinsäure zu diskutieren übrig.

1) Neubauer u. Flatow, Diese Zeitschrift, Bd. VII, S. 375.

2) Journ. of Physiol., Bd. XXXVI, 29. Nov. 1907. 
Von ihr ist nachgewiesen, daß der Normale sie zu verbrennen vermag, und dadurch ist wahrscheinlich gemacht, daß sie ein physiologisches Zwischenprodukt der Tyrosin- und Phenylalaninverbrennung ist, und daß die Abnormität der Alkaptonurie keine Abweichung von der normalen Oxydation dieser Aminosäuren darstellt, sondern einen Hemmungsvorgang in der Oxydation. Homogentisinsäure ist als normales Zwischenprodukt der Eiweißverbrennung zu betrachten.

Als solches hat die Homogentisinsäure von zahlreichen Bearbeitern Beachtung gefunden und das Studium der quantitativen Alkaptonausscheidung nach Verfütterungen sollte schließlich das erste Licht in das Dunkel des Aminosäureabbaus werfen.

Denn an sich läßt sehr weitgehende Schlüsse über den Mechanismus des Tyrosin- und Phenylalaninabbaus die Tatsache der Homogentisinsäureausscheidung allein nicht zu.

Auch abgesehen von den Veränderungen am Benzolkerne, die hier einer eingehenderen Betrachtung nicht unterzogen werden sollen, stellt die Homogentisinsäure bereits eine ziemlich fortgeschrittene Phase des Abbaus dar; die ersten Stadien der Oxydation, vor allem die Frage der Desaminierung erklärt die Alkaptonurie als solche nicht.

Man war bisher geneigt, den Vorgang der Desaminierung von Aminosäuren als einen hydrolytischen Prozeß anzunehmen; darauf weisen schon die Konstitutionsdeutungen hin, die unbekannten Körpern im Harne zuteil wurden. (Blendermanns p-Oxyphenylmilchsäure, Kirks Uroleucinsäure usw.).

Die Vorstellung, daß durch hydrolytische Prozesse primär Aminosäuren in Oxysäuren und Ammoniak übergehen möchten, konnte auch wohl dadurch eine höhere Wahrscheinlichkeit beanspruchen, als ähnliche Vorgänge in der Puringruppe festgestellt wurden, wo Adenin in Hypoxanthin und Guanin in Xanthin fermentativ überführbar sind.

$$
\underset{\text { Adenin. }}{\mathrm{H}_{2} \mathrm{O}+\underset{\left.\mathrm{C}_{6} \mathrm{H}_{3} \mathrm{C}_{4}\right) \mathrm{NH}_{2}}{\longrightarrow} \underset{\text { Hypoxanthin. }}{\left(\mathrm{C}_{6} \mathrm{H}_{8} \mathrm{~N}_{4}\right) \cdot \mathrm{OH}}+\mathrm{NH}_{3} .}
$$

Im Gegensatz zu dieser Annahme und bei ihrer Prüfung fand aber Neubauer, daß p-Oxyphenylmilchsäure, also ein "desaminiertes Tyrosin» bei Verabreichung an einen Alkapton- 
Über den Abbau von Aminosăuren im Organismus.

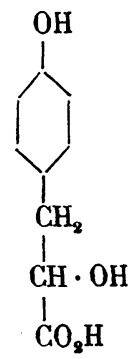

uriker keine Steigerung der Alkaptonausscheidung bewirkte. Die Verbindung konnte also unmöglich ein Zwischenprodukt des Tyrosinabbaus sein.

Dagegen bewirkte Verabreichung von p-Oxyphenylbrenztraubensäure<smiles>O=C(O)CC1CCC(O)CC1</smiles>

einen starken Anstieg der Homogentisinsäureausscheidung.

Diese Substanz war also eher das physiologische Zwischenprodukt und Neubauer schloB aus diesen beiden Resultaten, daß nicht die Oxysäuren, sondern die Ketonsäuren den Aminosäuren den Weg der Oxydation vorzeichnen.

Um diesen Vorstellungen eine weitere Stütze zu geben, untersuchte er an einer körperfremden Aminosäure, der Phenylaminoessigsäure,

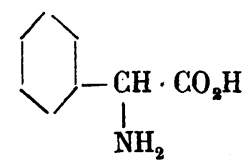

ob und auf welche Weise der Organismus sich der Aminogruppe entledigt.

Er fand, daß der Hund nach Fütterung mit dieser Säure nur deren Rechts-Anteil oxydativ angreift. Die l-Phenylaminoessigsäure erscheint unverändert im Harne. Der Rechts-Anteil 
aber wird als Phenylglyoxylsäure

zur Ausscheidung gebracht.

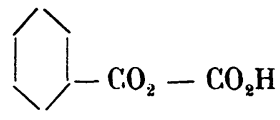

Beim Kaninchen findet - sekundär, wie Neubauer feststellte - eine teilweise Reduktion der Ketonsäure zu der ihr entsprechenden l-Mandelsäure statt.

Durch Neubauers Untersuchungen ist den $\alpha$-Ketonsäuren eine gleiche Bedeutung für den Eiweißst offwechsel zuteil geworden, wie sie die $\beta$-Ketonsäuren seit Erkenntnis der Fettverbrennung beanspruchen.

Es handelte sich nunmehr darum, diese Erfahrungen auf eine breitere Basis zu stellen und insbesondere auch für andere Aminosäuren den Beweis zu erbringen, daß sie im Organismus in Ketonsäuren überzugehen vermögen.

Die Phenylamidoessigsäure, die Neubauer verfütterte, war vielleicht deshalb nicht einmal ein besonders günstiges Studienmaterial, weil sie über die Ketonsäure hinaus nicht verbrannt werden kann. Das war für die Auffindung der Phenylglyoxylsäure «war günstig, physiologisch noch beweisender hätte indessen die Verfütterung körperfremder Aminosäuren mit dem Alaninrest als Seitenkette sich vielleicht gestalten können.

Aus folgendem Grunde:

Blum (Arch. f. exp. Pathol. u. Pharm. 1908) hatte feststellen können, daß o-Tyrosin und m-Tyrosin

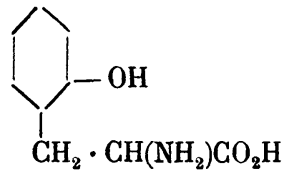

und

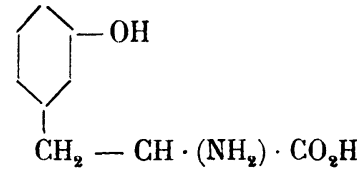

bei ihrer Verfütterung die ihnen entsprechenden Phenylessigsäuren liefern:

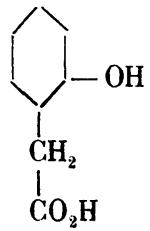

und

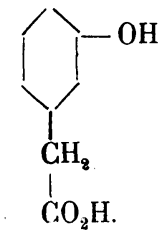


Das ist deshalb von außerordentlicher Bedeutung, weil damit eine weitgehende Analogie mit der Entstehung der Homogentisinsäure aus Tyrosin gegeben ist - also mit einem physiologischen Vorgange.

Würde nun hier oder in ähnlichen Fällen, wo der Alaninrest zum Essigsäurerest oxydiert wird, das Auftreten von Ketonsäuren als intermediäres Produkt zwischen Aminosäuren und Essigsäuren nachweisbar sein, so wäre damit die physiologische Bedeutung der Ketonsäuren noch klarer demonstriert, wie durch die ausschließliche und definitive Bildung von Phenylglyoxylsäure aus der unphysiologischen Glycylseitenkette.

Mit Genehmigung von Herrn Dr. Neubauer und mit seiner liebenswürdigen Unterstützung habe ich mich deshalb der Aufgabe unterzogen:

1. Die Verfütterungsversuche Blums mit 0 - und $m$-Tyrosin bei gleichzeitiger Berücksichtigung eventuell entstehender $\alpha$-Ketonsäuren $\mathrm{zu}$ wiederholen und entsprechend $\mathrm{zu}$ vertiefen,

2. m-Chlorphenylalanin - bisher unbekannt - zu synthetisieren und zu verfüttern, und schließlich

3. im Harne von einem Alkaptonuriker auf Ketonsäuren zu fahnden.

Im Anschluß an 1. und 2. möchte ich ferner über Versuche berichten, die das von Herrn Dr. Fischer und mir gemeinsam synthetisierte und verfütterte Furylalanin betreffen.

\section{Praktisoher Teil.}

Verfütterungsversuche mit 0 -Tyrosin.

Das von L. Blum synthetisch erhaltene o-Tyrosin (Arch. f. exp. Pathol. u. Pharm. 1908, S. 290) wurde in einer Menge von $3 \mathrm{~g}$ an ein Kaninchen in 2 Portionen mit Schlundsonde verabfolgt. Der 12 Stunden nach der letzten Eingabe mit Katheter entleerte Harn war infolge gleichzeitig dargereichten Natriumbicarbonates alkalisch. .

Er wurde im Vakuum bei $40^{\circ}$ eingeengt, angesäuert und mit Äther wiederholt extrahiert. 
Der Atherrückstand gab mit $\mathrm{FeCl}_{3}$ eine violette Färbung.

Es wurde in folgender Weise auf die eventuell vorhandene 0-0xyphenylbrenztraubensäure gefahndet.

Der Ätherrückstand wurde mit $10 \mathrm{ccm} 20 \%$ iger $\mathrm{NaOH}$ 20 Minuten lang erhitzt, um das möglicherweise beim Ansäuern des Harnes entstandene Lacton<smiles>O=C1CC2CCCCC2O1</smiles>

zur Aufspaltung in die freie Säure zu bringen. Der klaren Lösung wurden $20 \mathrm{ccm}$ Natriumbisulfitlauge zugesetzt und darauf unter Kühlung vorsichtig $5 \mathrm{ccm} \mathrm{HCl}$ (sp. G. 1,19).

Darauf wurde mit Äther extrahiert, der vorher mit schwefliger Säure gesättigt worden war. Bei diesem Verfahren, das jedwede Ketonsäure von anderen ätherlöslichen Säuren zu isolieren gestattet, bleibt die Ketonsäure beim Bisulfit, die o-Oxyphenylessigsäure (die Blum bereits - wie oben erwähnt - isoliert hat) im Äther.

Erst nach dem Ansäuern und Erwärmen der Bisulfitlösung geht bei der Extraktion mit Äther die Ketonsäure, falls vorhanden, in diesen über.

Bei der eben zitierten Versuchsanordnung gelang die Auffindung der o-Oxyphenylbrenztraubensäure im Kaninchenharn nicht.

Eine Kontrollprobe mit synthetisch dargestellter und dem Urin zugesetzter 0-Oxyphenylbrenztraubensäure ließ aber kleinste Spuren derselben nach der Trennung mittels Bisulfit durch die charakteristische $\mathrm{FeCl}_{3}$-Reaktion (blaugrün) erkennen.

Die 0-0xyphenylbrenztraubensäure scheint also sogleich weiter zu o-0xyphenylessigsäure oxydiert worden $z u$ sein, die auch in reichlicher Menge tatsächlich isoliert werden konnte. War diese Vorstellung richtig, so mußte verfütterte $0-0 x y-$ phenylbrenztraubensäure ebenfalls in 0-Oxyphenylessigsäure übergehen. 
Verfütterung von 0-0xyphenylbrenztraubensäure

$$
-\mathrm{CH}_{2}-\mathrm{CO}-\mathrm{CO}_{2} \mathrm{H} \text { als Lacton. }
$$

Die freie o-Oxyphenylbrenztraubensäure entsteht aus dem von Ploechl und Wolfrum (Ber. d. Deutsch. Chem. Ges., Bd. XVIII, S. 1183) erhaltenen o-Oxybenzoyliminozimmtsäurelactimid durch Kochen mit $\mathrm{NaOH}$.

Ihre Eigenschaften sind bekannt, vor allem, daß sie leicht in ihr Anhydrid, das Oxycumarin<smiles>O=C1OC2CCCCC2CC1(O)O</smiles>

übergeht, das seinerseits durch Alkalien wieder zur freien Säure aufgespalten werden kann.

Neubauer hat diese Substanz (das Lacton) bereits an einen Alkaptonuriker verabreicht, ohne daß dieser eine Steigerung seiner Alkaptonausscheidung erfahren hätte.

Er hatte, ebenso wie ich bei dem nachfolgenden Versuche, bei der Verabreichung dieses Lactons angenommen, daß es im Organismus zunächst eine hydrolytische Aufspaltung erfahren würde. Das ist indessen nicht der Fall, wie folgender Versuch zeigt.

$5,6 \mathrm{~g}$ des Oxycumarins wurden in 3 Portionen einem Manne (körperlich gesunder Neurastheniker) eingegeben. Gleichzeitig Natriumbicarbonat.

Harn frei von Eiweiß, zeigt Nachtrommer.

Beim Ansäuern des nicht einmal konzentrierten Harnes mit $\mathrm{H}_{2} \mathrm{SO}_{4}$ fallen $6,8 \mathrm{~g}$ einer in Nadeln krystallisierten, schönen weißen Verbindung langsam aus. Diese zeigt keine Färbung mit $\mathrm{FeCl}_{3}$, reduziert bei kürzerem Erwärmen alkalische Kupferlösung nicht.

Sie ist stark optisch aktiv. Die aus heißem Wasser umkrystallisierte analysenfertige Substanz schmolz bei $207^{\circ}$ unter Zersetzung. $0,2664 \mathrm{~g}$ in $16 \mathrm{ccm}$ verdünnter $\mathrm{NaOH}$ drehten im $2 \mathrm{dm}$-Rohr $2,40^{\circ}$ nach links. 
L. Flatow,

Folglich spez. Drehung $=-72,05^{\circ}$. Es handelte sich demnach wohl um eine gepaarte Glukuronsäure. Das bestätigt der Versuch einer Spaltung durch HCl. Erwärmt man kurze Zeit die Verbindung mit $20 \%$ iger $\mathrm{HCl}$, so läßt sich durch Äther Oxycumarin extrahieren, während der Rückstand nach $\mathrm{Zu}$ fügung von $\mathrm{NaOH}$ Kupferlösung stark reduziert.

Die Analyse stimmt gleichfalls auf die Formel einer

Anhydro-oxyphenylbrenztraubenglukuronsäure.

Substanz erst im Vakuum, dann bei $150^{\circ}$ zur Konstanz getrocknet.

$0,1057 \mathrm{~g}$ ergaben $0,2059 \mathrm{~g} \mathrm{CO}_{2}$ und $0,0416 \mathrm{~g} \mathrm{H}_{2} \mathrm{O}$.

Berechnet für $\mathrm{C}_{15} \mathrm{H}_{14} \mathrm{O}_{9}: \mathrm{C}=53,25 \%, \mathrm{H}=4,48 \%$.

Gefunden: $\mathrm{C}=53,12 \%, \mathrm{H}=4,39 \%$.

Andere ätherlösliche Substanzen, die auf das verfütterte Material zu beziehen waren, konnten im Harne nicht nachgewiesen werden.

Verfütterung der freien $0-0 x y$ phenylbrenztraubensäure.

Da der Ringschluß offenbar für den Organismus zu fest ist, als daß er im Kreislauf gelöst werden kann, wurde die freie o-0xyphenylbrenztraubensäure als Na-Salz an ein Kaninchen verabreicht. In folgender Weise: Das Anhydrid wurde einige Zeit mit 10\% iger Natronlauge (etwas mehr als die theoretische Menge) gekocht. Dann wurde bis zur völligen Sättigung $\mathrm{CO}_{2}$ eingeleitet und diese Lösung in 2 Portionen binnen 12 Stunden per Schlundsonde eingegeben. Die Menge entsprach 5,0 g o-Oxyphenylbrenztraubensäure.

Der ausgeschiedene Harn wurde verarbeitet, wie beim o-Tyrosinversuch angegeben.

So wurden $0,9 \mathrm{~g}$ unveränderter Ketonsäure als Anhydrid wieder gewonnen, neben $1,3 \mathrm{~g}$ o-Oxyphenylessigsäure. Die Reindarstellung der beiden Substanzen gelang durch die Bisulfittrennungsmethode ausgezeichnet. Zur Identifizierung und Analyse war für die Oxyphenylessigsäure einfaches Umkrystallisieren aus siedendem Wasser erforderlich. - Eine Umwandlung in das Lacton, Schmelzpunkt $49^{\circ}$, durch Destillation wurde zur Identi- 
fizierung mit einer Probe vorgenommen (A.Blum), zwecks Reindarstellung genügte aber die Krystallisation. - Schmelzpunkt $137^{\circ}$.

Analyse: 0,115 g Substanz lieferten $\mathrm{CO}_{\mathbf{z}}=0,2674 \mathrm{~g}$ $\mathrm{H}_{8} \mathrm{O}=0,0561$.

Berechnet für $\mathrm{C}_{8} \mathrm{H}_{8} \mathrm{O}_{8}: \mathrm{C}=63,15 \%, \mathrm{H}=5,28 \%$.

Gefunden: $\mathrm{C}=63,22 \%, \mathrm{H}=5,4 \%$.

Die 0-0xyphenyltraubensäure war also im Gegensatze $\mathrm{zu}$ ihrem Lacton im Organismus in 0-0xyphenylessigsäure umgewandelt worden.

\section{Verfütterung von m-Tyrosin.}

Nach Eingabe von allerdings geringen Mengen o-Tyrosins war mir der direkte Nachweis von 0-0xyphenylbrenztraubensäure im Harne nicht gelungen.

Ich versuchte deshalb - und dabei mit etwas besserem Erfolge - den Nachweis von m-Oxyphenylbrenztraubensäure im Harne nach Verabreichung von m-Tyrosin.

m-Tyrosin wurde nach Blums Angaben(loc. cit.) gewonnen.

$\check{\mathrm{g}} \mathrm{g}$ hiervon wurden gemischt mit Natriumbicarbonatlösung einem Kaninchen mittels Schlundsonde beigebracht. (2 Portionen binnen 12 Stunden.)

Der darauf ausgeschiedene Harn wurde im Vakuum eingeengt und außerordentlich gründlich nach dem Ansäuern mit Äther extrahiert.

[Die nachfolgend beschriebene synthetische Darstellung der eventuell zu isolierenden $m$-Oxyphenylbrenztraubensäure hatte nämlich gelehrt, daß diese Säure, trotzdem sie sowohl in Wasser wie auch in Äther sehr gut löslich ist, dennoch schwer in guter Ausbeute durch Äther aus wässeriger Lösung ausgeschüttelt wird. (Gleichgewichtszustand eines schwer ätherlöslichen Hydrates??)]

Der Ätherrückstand wurde mit Natriumbisulfitlauge aufgenommen und letztere wiederum mit $\mathrm{SO}_{2}$-gesättigtem Äther extrahiert. In dieses Ätherextrakt geht quantitativ die entstandene $\mathrm{m}$-0xyphenylessigsäure $(1,4 \mathrm{~g})$. Die Bisulfitlauge hält die m-0xyphenylbrenztraubensäure zurück. Man übersättigt sie darauf mit Salzsäure bis zur dauernden Kongoreaktion und erhitzt kurze Zeit auf dem Wasserbade unter Luftdurchleiten. 
Dann wird nach erfolgter Abkühlung der Flüssigkeit die Ketonsäure durch Äther entzogen.

Auch hierbei ist häufiges Extrahieren erforderlich.

Der Ätherrückstand enthielt in meinem Versuch sehr geringe Mengen $(0,06 \mathrm{~g})$ einer Substanz, die sich leicht in Wasser löste, deren Lösung intensive Blaugrünfärbung mit $\mathrm{FeCl}_{3}$ gab, die ein krystallisierendes Phenylhydrazon mit salzsaurem Phenylhydrazon lieferte, die bei $155^{\circ}$ nach vorhergegangener Zersetzung schmolz; kurz, die sämtliche Eigenschaften der gleich zu beschreibenden synthetisch erhaltenen m-Oxyphenylbrenztraubensäure zeigte, und die auch mit ihr gemischt den gleichen Schmelzpunkt behielt.

Auch bei Blums Versuchen scheint diese Säure vorhanden gewesen zu sein. Er verfütterte $5 \mathrm{~g}$ m-Tyrosin und isolierte 1,2 $\mathrm{g}$ der $\mathrm{m}$-Phenylessigsäure, die keine charakteristische Eisenchloridfärbung gibt. Blum führt aber ausdrücklich in seinem Protokoll an, daß das Ätherextrakt des Harnes stark blaugrüne $\mathrm{FeCl}_{3}$-Reaktion zeigte.

\section{Darstellung und Verfütterung von m-Oxyphenyl- brenztraubensäure.}

Wenn man das Kondensationsprodukt von m-Oxybenzaldehyd und Hippursäure (cf. Blum) mit 20\% iger Natronlauge bis zum Aufhören der $\mathrm{NH}_{3}$-Entwickelung kocht, dann ansäuert und nach dem Ausfallen der Benzoesäure die Lösung sehr häufig mit Äther extrahiert, erhält man die m-Oxyphenylbrenztraubensäure. Sie enthält etwas Benzoesäure und etwas Essigsäure, letztere wegen der Verseifung der ursprünglich im Molekül vorhanden gewesenen Äthylgruppe.

Über Natronkalk geht die Essigsäure fort. Die Verunreinigung mit Benzoesäure wird am besten durch Reinigung mittels der schon genügend erwähnten Bisulfitmethode beseitigt.

Für Analysenzwecke diente ein Präparat, das Herr Dr. Neubauer bereits vor einem Jahre hergestellt hatte. Dasselbe wurde aus Benzol (十 Äther) mittels Petroläther als sehr elektrischer und hygroskopischer Niederschlag erhalten und wiederholt umgefällt. 
Nach Konstanztrocknung über Paraffin im Vakuum wurden folgende Analysenwerte erhalten:

$0,1061 \mathrm{~g}$ Substanz ergaben $0,2374 \mathrm{~g} \mathrm{CO}_{\mathbf{2}}$ und $0,0465 \mathrm{~g} \mathrm{H}_{\mathbf{2}} \mathrm{O}$.

Berechnet für $\mathrm{C}_{8} \mathrm{H}_{8} \mathrm{O}_{4}: \mathrm{C}=60,00 \%, \mathrm{H}=4,44 \%$.

Gefunden: $\mathrm{C}=61,10 \%, \mathrm{H}=4,9 \%$.

Obgleich die Analysenwerte wohl infolge des Krystallisationsmittels nicht gerade befriedigen, kommt doch nach Entstehung und Eigenschaften für die Verbindung keine andere Formel in Betracht als die einer m-0xyphenylbrenztraubensäure.

Zur Verfütterung war ein Präparat frischer Darstellung verwendet worden. Verabreicht wurden $4,8 \mathrm{~g}$ in Natriumbicarbonatlösung an ein Kaninchen (Schlundsonde).

Der ausgeschiedene Harn wurde im Vakuum bei $40^{\circ}$ eingeengt, und, wie oben beschrieben, nach dem Bisulfitverfahren verarbeitet.

Auf Isolierung geringer Mengen noch vorhandener Ketonsäure wurde kein Gewicht gelegt.

Es wurden $0,9 \mathrm{~g} \mathrm{~m}$-0xyphenylessigsäure gewonnen, die aus Benzol umkrystallisiert den Schmelzpunkt 128,5 (unkorr.) zeigte. Analyse: $0,1540 \mathrm{~g}$ Substanz lieferten $0,3565 \mathrm{~g} \mathrm{CO}_{2}$ und $0,0748 \mathrm{~g} \mathrm{H}_{\mathbf{2}} \mathrm{O}$.

Berechnet für $\mathrm{C}_{8} \mathrm{H}_{8} \mathrm{O}_{8}: \mathrm{C}=62,76 \%, \mathrm{H}=5,22 \%$.

$$
\text { Gefunden: } \mathrm{C}=63,06 \%, \mathrm{H}=5,4 \% \text {. }
$$

Bei Verfütterung von $m$-Tyrosin war es also möglich, sowohl intermediär gebildete Ketonsäure in Substanz nachzuweisen, als auch zu zeigen, daß die Ketonsäure verfüttert in $\mathrm{m}$-Oxyphenylessigsäure übergeht, ebenso wie das $\mathrm{m}$-Tyrosin zum größten Teile selbst.

Darstellung und Verfütterung von m-Chlorphenylalanin.

Die Synthese des bisher unbekannten m-Chlorphenylalanins ist völlig analog der des Phenylalanins selbst zu vollziehen.

m-Chlorbenzoylamidozimmtsäurelactimid.

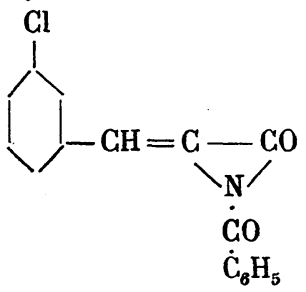


Man kondensiert zu seiner Herstellung 1 Molekül m-Chlorbenzaldehyd mit 1 Molekül Hippursäure bei Gegenwart von 1 Molekül wasserfreiem Natriumacetat und 11/2 Molekülen Essigsäureanhydrid. Die Reaktion der gut gemischten Komponenten ist auf siedendem Wasserbade in $1 / 2$ Stunde beendet. Das Reaktionsprodukt stellt einen gelben Krystallbrei dar, der mit großen Mengen Wassers bis zur völligen Zersetzung des überschüssigen Essigsäureanhydrids gekocht wird. Man saugt vom Gelösten ab, wäsche den gelben Rückstand mit Alkohol, dann mit Äther und besitzt nach dem Trocknen in dem erhaltenen Produkte einen für weitere Verarbeitung hinreichend reinen Körper. Zur Analyse wurde aus Eisessig umkrystallisiert:

Ausbeute $75 \%$ der Theorie:

$0,2557 \mathrm{~g}$ Substanz lieferten nach Kjeldahl eine Menge $\mathrm{NH}_{3}$, welche

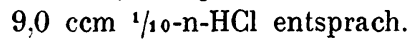

$$
\text { Berechnet für } \begin{aligned}
\mathrm{C}_{16} \mathrm{H}_{10} \mathrm{NO}_{2} \mathrm{Cl}: \mathrm{N} & =4,94 \% \% . \\
\text { Gefunden : } \mathrm{N} & =4,93 \% .
\end{aligned}
$$

In der Kälte ist die Verbindung in verdünnten Alkalien unlöslich, beim Erhitzen löst sie sich in einer 3\% igen Lösung der theoretisch zur Aufspaltung erforderlichen Alkalimenge(1Mol.). Das Aufspaltungsprodukt, die Benzoylamido-m-Chlorzimmtsäure, ist durch Säuren aus der alkalischen Lösung ausfällbar. Sie wurde aber als unwesentlich nicht weiter untersucht.

Zur Gewinnung des Benzoyl-m-Chlorphenylalanins wurde vielmehr stets von einer genau abgewogenen Menge des gelben Kondensationsproduktes aus Hippursäure und mChlorbenzaldehyd ausgegangen.

Dieses wurde zur besseren Benetzbarkeit mit Alkohol angefeuchtet und mit einer $6 \%$ igen Natronlauge (theoretische Menge) unter Erwärmen auf dem Wasserbade zur Lösung gebracht. Nach dem Filtrieren und Abkühlen wurde in dickwandiger Schüttelflasche mit der genau berechneten Menge $2,3 \%$ iger Natriumamalgams reduziert. (Es ist erforderlich, daß das Natriumamalgam frisch hergestellt und sein Gehalt an Natrium genau titrimetrisch bestimmt wird.)

Dabei geht das Natriumsalz der Benzoylamido-m-Chlorzimmtsäure in das des Benzoylchlorphenylalanins über. 
Über den Abbau von Aminosäuren im Organismus.

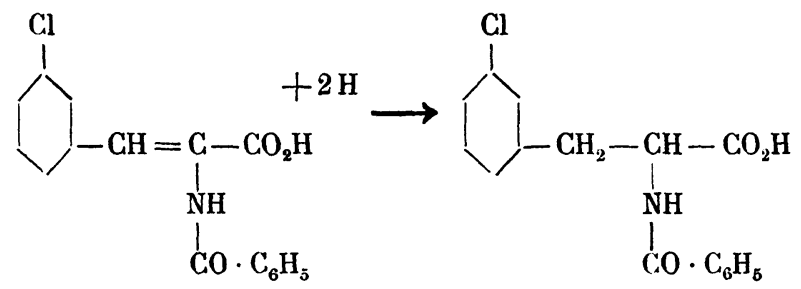

Verwendet man aber mehr als die theoretische Menge Natriumamalgams, so besteht die Gefahr, daß Chlor aus dem Kerne herausgerissen wird, was indessen erst dann geschieht, wenn die doppelte Bindung in der Seitenkette gelöst ist.

- Nach erfolgter Reduktion wird mit rauchender Salzsäure bis zur Reaktion auf Kongopapier angesäuert, wobei das Benzoyl-m-Chlorphenylalanin zunächst ölig, dann aber bald krystallinisch erstarrend ausfällt.

Die Ausbeute ist quantitativ. Zur Analyse wurde aus Eisessig umkrystallisiert. Rosettenförmig angeordnete Säulen mit aufgesetzter Pyramide vom Schmelzpunkt $174^{\circ}$ (unkorr.)

$0,2023 \mathrm{~g}$ Substanz benötigen zur Neutralisation $6,7 \mathrm{ccm} \frac{\mathrm{NaOH}}{10} \mathrm{n}$.

Mol.-Gew. berechnet: 303,5; gefunden: 315 .

Die gleiche zur Titration verwendete Lösung wurde in einem Kjeldahlkolben einer $\mathrm{N}$-Bestimmung unterzogen.

Erhalten wurde $\mathrm{NH}_{3}$, entsprechend $6,7 \mathrm{~cm} 1 / 10-\mathrm{n}-\mathrm{HCl}$.

Berechnet für die Säure: $N=4,61 \%$.

Gefunden: $\quad \mathrm{N}=4,63 \%$.

Das Benzoyl-m-Chlorphenylalanin ist in Wasser außerordentlich schwer löslich, desgleichen in Salzsäure. Deshalb benötigt man $\mathrm{zu}$ seiner Spaltung in $\mathrm{m}$-Chlorphenylalanin<smiles>NC(CC1CCCC(Cl)C1)C(=O)O</smiles>

recht beträchtliche Mengen Salzsäure. 1 Teil bedarf etwa 100 Teile Salzsäure 1,125. Trotzdem geht die Lösung sehr langsam. Nach 4-5 Stunden ist noch ein großer Teil ungelöst.

Im ganzen pflegte ich 20 Stunden ununterbrochen zu kochen. 
Dann ist die Zersetzung quantitativ. Man engt ein und erhält ein Gemisch von salzsaurem Chlorphenylalanin, welches in Salzsäure sehr schwer löslich ist, und von Benzoesäure. Letztere wird mittels Äthers entfernt.

Das salzsaure Salz, welches mit dem des Phenylalanins sehr große Ähnlichkeit hat, silberglänzende Prismen, verliert an der Luft teilweise Salzsäure. Es wurde deshalb nur das freie Chlorphenylalanin analysiert. Dieses entsteht aus der konzentrierten Lösung des salzsauren Salzes durch Fällung mit Nàtriumbicarbonat. - Das Chlorphenylalanin ist in Wasser in der Kälte schwer, beim Erhitzen leichter löslich. Seine wässerige Lösung schmeckt zunächst süß, dann widerlich bitter. - Farblose zu Häufchen gruppierte Nadeln. Schmelzpunkt $234^{\circ}$ u. Z.

$\mathrm{N}$-Bestimmung nach Kjeldahl:

$0,2210 \mathrm{~g}$ lieferten $\mathrm{NH}_{3}, 11,3 \mathrm{ccm} 1 / 10-\mathrm{n}-\mathrm{HCl}$ entsprechend.

Berechnet für $\mathrm{C}_{9} \mathrm{H}_{10} \mathrm{O}_{2} \mathrm{NCl}=7,02 \%$.

Gefunden: $\quad=7,16 \%$.

Verfütterung von Chlorphenylalanin an Kaninchen.

Verabreicht wurden in Bicarbonatlösung im Laufe von 3 Tagen $14 \mathrm{~g}$ Chlorphenylalanin. (Schlundsonde.)

Der gesammelte Urin wurde im Vakuum eingeengt, angesäuert mit $\mathrm{HCl}$ und mit Äther wiederholt ausgeschüttelt. Der Ätherrückstand zeigte die für alle Phenylbrenztraubensäuren so charakteristische intensiv blaugrüne $\mathrm{FeCl}_{3}$-Reaktion.

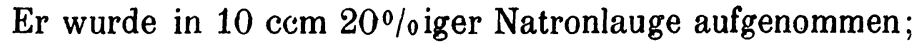
hierzu wurden $100 \mathrm{ccm}$ Bisulfitlauge und dann $5 \mathrm{~cm}$ rauchende Salzsäure (1,19 spez. Gew.) gegeben. - Extraktion mit $\mathrm{SO}_{2^{-}}$ gesättigtem Äther. (Extrakt A.)

Die Bisulfitlösung wurde dann stark mit Salzsäure angesäuert, erwärmt, und die schweflige Säure mittels Luft ausgetrieben. Darauf Extraktion mit Äther. (Extrakt B.)

Das Extrakt B lieferte beim Verdampfen Krystalle, die aus Wasser umkrystallisiert den Schmelzpunkt und die Eigenschaften der synthetisch (s. u.) erhaltenen m-Chlorphenylbrenztraubensäure zeigten. Zur Analyse habe ich die ganze Menge in das gut charakterisierte Phenylhydrazon übergeführt. Es 
wurde der Rückstand in heißem Wasser gelöst, filtriert und mit einer filtrierten Lösung von salzsaurem Phenylhydrazin versetzt. Gelbe Nadeln. (2,6 g!) Umkrystallisiert aus Eisessig (unter Zusatz von Tierkohle) + Wasser. Schmelzpunkt des Phenylhydrazons $159^{\circ}$.

$0,1090 \mathrm{~g}$ Substanz ergaben $9,2 \mathrm{ccm} \mathrm{N}$ bei $14^{\circ}$ und $724 \mathrm{~mm}$.

$\begin{aligned} \text { Berechnet für } \mathrm{C}_{15} \mathrm{H}_{13} \mathrm{~N}_{8} \mathrm{O}_{2} \mathrm{Cl} & =9,73 \% \mathrm{~N} . \\ \text { Gefunden } & =9,56 \%\end{aligned}$

Man kann das Phenylhydrazon auch direkt aus der Bisulfitlösung durch Zusatz von Phenylhydrazinchlorhydrat in wenig Salzsäure durch Erwärmen fällen.

Der Ätherrückstand A enthält gleichfalls eine Substanz oder deren mehrere, die Chlor in organischer Bindung enthalten, somit in Beziehung zum Abbau des Chlorphenylalanins stehen. - Wahrscheinlich liegt der Hauptmenge nach die mChlorphenylessigsäure vor; daneben aber wohl auch die Chlorphenylmilchsäure in geringer Menge, denn es konnte eine Linksdrehung von $0,25^{\circ}$ festgestellt werden, die auch bei der weiteren Verarbeitung des Rückstandes erhalten blieb.

Um nämlich Hippursäure und eine etwa entstandene Chlorphenacetursäure (Phenylessigsäure liefert nach Verfütterung Phenacetursäure) aus dem Ätherrückstand auszuschalten, wurde dieser mit siedendem Benzol wiederholt ausgekocht, da die Erfahrung lehrt, daß auf diese Weise die Glykokollpaarlinge im unlöslichen Rückstand zu verbleiben pflegen.

Tatsächlich war auch nach dem Verdampfen des Benzols der Rückstand stickstofffrei. - Er drehte aber noch immer $0,22^{\circ}$ links.

Die verschiedenen Versuche, die hier vorliegenden Substanzen rein darzustellen, waren sämtlich erfolglos.

Zwar läßt sich durch Lösen des Benzolrückstandes in wenig siedendem Wasser und Kochen mit gefälltem Calciumcarbonat ein in kaltem Wasser schwerlösliches und prächtig großkrystallisierendes Kalksalz gewinnen; aber bei der Umsetzung mittels Säuren wurde immer wieder ein Öl erhalten. Die Menge desselben war auch in jedem Falle so gering, daß eine weitere Reinigung aussichtslos erschien. - Die Mutter-

Hoppe-Seyler's Zeitschrift f. physiol. Chemie. LXIV. 
lauge des Kalksalzes enthielt die optisch linksdrehende Substanz. Das schwerlösliche Kalksalz selbst war optisch inaktiv. Es bleibt über die Versuche mit m-Chlorphenylalanin nur noch zu berichten, daß die gleichen Produkte wie bei seiner Verfütterung auch durch Verfütterung von m-Chlorphenylbrenztraubensäure (synthetisch) erhalten werden.

Die m-Chlorphenylbrenztraubensäure entsteht quantitativ durch Spaltung des Kondensationsproduktes von m-Chlorbenzaldehyd mit Hippursäure durch siedende 30\%ige Natronlauge. Durch Anwendung der Bisulfitmethode ist eine Trennung von gleichzeitig entstandener Benzoesäure leicht zu bewerkstelligen. Man kocht also z. B. $30 \mathrm{~g}$ des Kondensationsproduktes mit $250 \mathrm{ccm} 40 \%$ iger Natronlauge und $70 \mathrm{ccm} W$ asser, bis die $\mathrm{NH}_{3}$-Entwicklung aufhört, fügt $1 / 2$ Liter Bisulfitlauge hinzu und dazu nach erfolgter Kühlung $250 \mathrm{ccm} \mathrm{HCl}$ (sp. G. 1,19). Es fällt nur Benzoesäure aus. Erst nach dem Übersäuern(Kongo!) mit $\mathrm{HCl}$ und Erhitzen unter Luftdurchleiten fällt die m-Chlorphenylbrenztraubensäure als schneeweißer, krystallisierter Niederschlag von den Eigenschaften, die oben gelegentlich der Verfütterung von m-Chlorphenylalanin für sie angegeben wurden, aus. Kleinere Mengen werden noch durch Extraktion mit Äther aus der Mutterlauge gewonnen.

Verfütterung von m-Chlorphenylmilchsäure.<smiles>O=C(O)C(O)CC1CCCC(Cl)C1</smiles>

Die Säure wird als eine in Blättchen vom Schmelzpunkt $125^{\circ}$ krystallisierende, farblose Verbindung durch Reduktion der Ketonsäure mit Natriumamalgam (theoretische Menge gleich 2 Atome) erhalten.

0,1606 g Substanz verbrauchten $8,05 \mathrm{ccm} 1 / 10-\mathrm{n}-\mathrm{NaOH}$.

Berechnet: Molekulargewicht 200,5

Gefunden:

199,5 . 
$1 \mathrm{~g}$ der Verbindung an ein Kaninchen verabreicht, bewirkte keine Grünfärbung des ausgeschiedenen Urins durch $\mathrm{FeCl}_{3}$. Folglich ist keine Ketonsäure entstanden und deshalb die Oxysäure nicht etwa als Vorstufe der Ketonsäure im Organismus zu betrachten (ebensowenig wie nach Neubauers Versuchen die Mandelsäure als Vorstufe der Phenylglyoxylsäure in Betracht kommen kann).

\section{Versuche mit Furylalanin.}

$$
{ }_{0}-\mathrm{CH}_{2}-\mathrm{CH}\left(\mathrm{NH}_{2}\right) \cdot \mathrm{CO}_{2} \mathrm{H} \text {. }
$$

Bevor die eben geschilderten Versuche mit $m$ - und o-Tyrosin und $\mathrm{m}$-Chlorphenylalanin ausgeführt wurden, haben Herr Dr. H. Fischer und ich gemeinsam das Furylalanin synthetisiert und dieses verfüttert, um dabei entstehende Ketonsäuren nachzuweisen. Die Verbindung erwies sich aber als so giftig, daß die Versuchstiere eingingen, bevor sie die für genauere Untersuchungen hinreichende Menge Harn produziert hatten. Die Sektion ergab stets starke Fettdegeneration der Leber.

Dennoch sollen die dabei neu hergestellten chemischen Individuen zum mindesten kurz angeführt werden.

Furylbenzoylaminoakrylsäurelactimid.

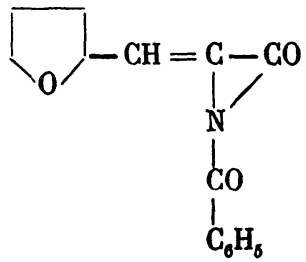

Durch Kondensation von Furfurol mit Hippursäure, Essigsäureanhydrid und Natriumacetat. Nadeln intensiv gelb, Schmelzpunkt (aus Eisessig umkrystallisiert) $170^{\circ}$. Ausbeute $70 \%$.

Wird durch $4 \%$ ige Natronlauge in die farblose freie Säure aufgespalten, die durch die theoretische Menge Natriumamalgam zum

Benzoylfurylalanin

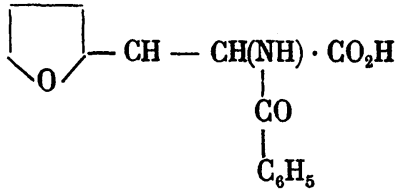


reduziert wird. Schmelzpunkt aus Wasser $163^{\circ}$. Rosetten aus Prismen bestehend. Das Furylalanin kann nicht wie in analogen Fällen durch Erhitzen seiner Benzoylverbindung mit Säuren erhalten werden. Die Empfindlichkeit des Furanringes bewirkt ein völliges Verharzen der unter dem Einflusse von Säuren sich bald rotbraun färbenden Lösung. Die Verseifung muß alkalisch geschehen.

$\mathrm{Zu}$ diesem Zwecke wird die Benzoylverbindung mit einem großen Überschuß 30\% $\mathrm{NH}_{3}$-Entwickelung gekocht. Dann wird die verdünnte Lösung sehr vorsichtig mit Schwefelsäure derart neutralisiert, daß gerade das neutrale Natriumsulfat entsteht. Kühlung. Nach dem Absaugen etwa ausgefallener Benzoesäure wird der Lösung schnell eine Lösung von überschüssigem Kupfersulfat zugesetzt und soviel Natronlauge unter Erhitzen auf dem Wasserbade, daß das Kupfersulfat dadurch noch nicht ganz quantitativ in Hydroxyd umgewandelt wird. Man engt nun ein bis zur Ausscheidung von Natriumsulfat, bringt dieses mit dem Kupfersalzniederschlag gemeinsam auf ein Filter, wäscht mit kaltem Wasser die löslichen Salze fort und zerlegt das meist mißfarbige Kupfersalz in heißer wässeriger Suspension mit $\mathrm{H}_{2} \mathrm{~S}$. Einige Tropfen Essigsäure, der Suspension zugesetzt, heben den kolloidalen Zustand auf, in dem sich oftmals das entstehende Schwefelkupfer auszuscheiden pflegt. Nach Filtration von letzterem wird das Filtrat im Vakuum zur Trockene eingeengt und das Furylalanin als Krystallkruste erhalten, die aus derben farblosen Prismen besteht. Man krystallisiert aus heißem Wasser um und erhält das Furylalanin als zunächst intensiv süß, darauf aber bitter schmeckende Substanz vom Schmelzpunkt oberhalb einer Temperatur von $240^{\circ}$. Reinigung eventuell über das Cu-Salz.

Das Furylalanin ist ausgezeichnet durch sein schwer lösliches $\mathrm{Cu}$-Salz, das indessen nicht durch essigsaures Kupfer aus Lösungen des Furylalanins gefällt werden kann, sondern nur durch direktes Auflösen frisch gefällten $\mathrm{Cu}$-Hydroxyds in siedender Furylalaninlösung. Am besten erhält man es - wie oben bei seiner Darstellung angegeben -, wenn man zu einer 
Furylalaninlösung (neutral) Kupfersulfat und eine letzterem entsprechende Menge Natronlauge in der Hitze zufügt.

Das Filtrat scheidet dann beim Abkühlen tief dunkelblaue Krystallaggregate des Cu-Salzes ab. Der Niederschlag von Cu-Hydroxyd enthält stets auch noch eine größere Menge des Furylalaninkupfers.

Das Furylalanin ergab bei einer $\mathrm{N}$-Bestimmung nach Kjeldahl folgende Analysenwerte.

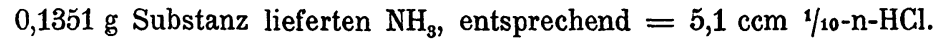

Berechnet: $\mathrm{N}=5,18 \%$,

Gefunden: $\mathrm{N}=\tilde{5}, 29 \%$.

Verfütterung von Furylalanin.

Gaben von ${ }^{1 / 2}-1 \mathrm{~g}$ waren durchschnittlich für ein Kaninchen binnen 36 Stunden tödlich. Der Urin dieser Tiere, sowie das Ätherextrakt derselben zeigte regelmäßig folgende Eisenchloridreaktion: "Ein Tropfen $\mathrm{FeCl}_{3}$ bewirkte intensiv violette Färbung, die sehr bald abblassend, einer schwach grünlichen gleichfalls vergänglichen wich.»

Das dürfte dem Verhalten einer Ketonsäure von der Konstitution einer Furylbrenztraubensäure

$$
\longrightarrow-\mathrm{CH}_{2}-\mathrm{CO} \cdot \mathrm{CO}_{2} \mathrm{H}
$$

wohl entsprechen, denn wenn man das Kondensationsprodukt von Furfurol und Hippursäure (s. S. 387) mit Natronlauge (30\%) kocht, die Lösung ansäuert und mit Äther extrahiert, zeigt der Ätherrückstand das gleiche Verhalten. Bei dieser Reaktion aber dürfte weitgehender Analogie zufolge die «Furylbrenztraubensäure» vorhanden sein.

Diese Versuche blieben wegen der Giftigkeit der verfütterten Substanz unvollendet. Jedenfalls legen sie mit großer Wahrscheinlichkeit auch hier die intermediäre Bildung von Ketonsäure aus einer Aminosäure mit heterocyklischem Ringe nahe.

Versuche zur Auffindung von Ketonsäuren im Harne eines Alkaptonurikers.

Von meinem Freunde, Herrn Dr. E. Magnus-Alsleben, erhielt ich aus Basel von dem gleichen Patienten, den Herr 
Dr. Neubauer eingehend wiederholt untersucht hat, Alkaptonurin übersandt. Im ganzen $2 \mathrm{l}$.

Ihm, sowie Herrn Prof. Gerhardt, dem Chef der Basler Klinik, gestatte ich mir für diese Liebenswürdigkeit meinen Dank auszusprechen.

Der angesäuerte Harn wurde im Vakuum zur Trockene eingedampft, der Rückstand mit Äther wiederholt ausgezogen und das Ätherextrakt in der folgenden Weise verarbeitet.

Im Wasserstoffstrom wurde es - um Bräunung zu vermeiden - mit $20 \mathrm{ccm}$ Natronlauge von $40 \%$ und $20 \mathrm{ccm}$ Wasser etwa eine halbe Stunde stehen gelassen. Dieses geschah in der Absicht, ein etwa vorhandenes Anhydrid der Hydrochinonbrenztraubensäure - dessen Eigenschaften durch die von 0 . Neubauer und mir vollzogene Synthese bekannt sind (l. c.) - und dem folgende Konstitution zukommt<smiles>O=C1C=CC2CCCC(O1)C2CO</smiles>

zur Hydrochinonbrenztraubensäure<smiles>O=C(O)CC1CC(O)CCC1O</smiles>

aufzuspalten, denn nur letztere gibt eine Bisulfitverbindung.

Nach $1 / 8$ stündigem Stehen im $\mathrm{H}$-Strome also wurden schnell um Luftoxydation zu vermeiden, der Lösung $100 \mathrm{ccm}$ Bisulfitlösung zugefügt und dann sofort unter Kühlung $19 \mathrm{ccm}$ rauchende Salzsäure vom spezifischen Gewicht 1,19. Es wurden dann zur Entfernung aller in Äther löslichen Säuren wiederholt Ausschüttelungen mit $\mathrm{SO}_{2}$-gesättigtem Äther $(10 \mathrm{mal})$ vorgenommen. In diesem fand sich die ganze Menge der Homogentisinsäure $(4,3 \mathrm{~g})$ neben Hippursäure und anderen ätherlöslichen Säuren.

Darauf wurde die bisulfithaltige Lösung mit $\mathrm{HCl}$ übersäuert, erwärmt und nach dem Erkalten gleichfalls wiederholt mit Äther extrahiert. Der Ätherrückstand war außerordentlich gering, kaum nennenswert. Mit Wasser aufgenommen zeigte 
er keine Spur einer $\mathrm{FeCl}_{3}$-Färbung und gab keine Trübung mit Phenylhydrazinchlorhydrat.

Es ist also in dem untersuchten Falle von Alkaptonurie das Vorhandensein von Ketonsäuren im Harne, insbesondere von Hydrochinonbrenztraubensäure auszuschließen.

\section{Zusammenfassung der Resultate.}

1. Sowohl o-Tyrosin wie o-Oxyphenylbrenztraubensäure werden vom Kaninchen zu o-Oxyphenylessigsäure abgebaut.

2. Das Lacton der o-Oxyphenylbrenztraubensäure wird im Organismus nicht aufgespalten, sondern mit Glukuronsäure gepaart.

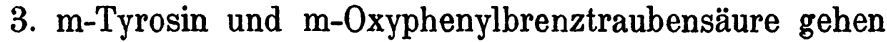
im Organismus in gleicher Weise in $m$-Oxyphenylessigsäure über. Bei Verfütterung von $m-T$ yrosin ist $m-0 x y p h e n y l-$ brenztraubensäure im Harne direkt nachweisbar.

4. Das bisher unbekannte $\mathrm{m}$-Chlorphenylalanin läßt nach Verfütterung reichlich m-Chlorphenylbrenztraubensäure im Harne erscheinen.

5. m-Chlorphenylmilchsäure wird nicht zur m-Chlorphenylbrenztraubensäure im Organismus oxydiert.

6. Das bisher unbekannte Furylalanin liefert nach Verfütterung eine nicht rein isolierte Substanz, welcher die Eigenschaften der auf anderem Wege erhaltenen Furylbrenztraubensäure zukommen.

7. Im Harne eines Alkaptonurikers konnte weder Hydrochinonbrenztraubensäure, noch überhaupt eine Ketonsäure nachgewiesen werden.

Die vorliegenden experimentellen Untersuchungen wurden im II. Mediz.-Klinischen Institut der Universität München aus-

Anmerkung: Nach völliger experimenteller Fertigstellung dieser Arbeit teilte Herr E. Friedmann (Medizin. Klinik, Jahrg. 5, S. 1402 [1909]) mit, daß er in Gemeinschaft mit Dr. Maase p-Chlorphenylalanin verfüttert habe.

Er kommt zu Resultaten, die ihn dazu führen, sich der Neubauerschen Vorstellung über den Abbau von Aminosäuren anzuschließen. 
392 L. Flatow, Über den Abbau von Aminosäuren im Organismus.

geführt. Dem Leiter desselben, Herrn Prof. Friedr. v. Müller, gestatte ich mir für seine stete Anteilnahme und die Förderung meiner Versuche meinen verbindlichsten Dank auszusprechen.

Herr Privatdozent Dr. 0. Neubauer hat mir in liebenswürdiger Weise gestattet, aus seinem speziellen Arbeitsgebiete das vorliegende Thema $\mathrm{zu}$ bearbeiten. Er hat mir mit Rat jederzeit zur Seite gestanden. Dafür ihm zu danken, ist mir eine angenehme Pflicht. 